\title{
A Novel Anti-cancer Strategy Targeting CD44-positive Cancer Stem Cells
}

\author{
Seishima $\mathbf{R}^{1,2}$, Okabayashi $K^{1 *}$, Nagano $O^{2}$, Hasegawa $\mathbf{H}^{1}$, Tsuruta $\mathbf{M}^{1}$, Saya $\mathbf{H}^{2}$ and Kitagawa $\mathbf{Y}^{1}$ \\ ${ }^{1}$ Department of Surgery, Keio University School of Medicine, 35 Shinano-machi, Shinjuku-ku, Tokyo 1608582, Japan \\ ${ }^{2}$ Division of Gene Regulation, Institute for Advanced Medical Research, Keio University School of Medicine, 35 Shinano-machi, Shinjuku-ku, Tokyo 1608582, Japan
}

${ }^{*}$ Corresponding author: Koji Okabayashi, Department of Surgery, Keio University School of Medicine 35 Shinano-machi, Shinjuku-ku, Tokyo, 1608582, Japan, Tel: +81 33353 1211; Fax: +81 33355 4707; E-mail: okabayashikoji@gmail.com

Received date: April 07, 2016; Accepted date: April 25, 2016; Published date: April 30, 2016

Copyright: (c) 2016 Okabayashi K, et al. This is an open-access article distributed under the terms of the Creative Commons Attribution License, which permits unrestricted use, distribution, and reproduction in any medium, provided the original author and source are credited.

\section{Introduction}

Despite progress in cancer therapy, cancer continues to be a common and lethal disease worldwide. Recent advances in cancer stem cell (CSC) research have indicated that CSCs, a subpopulation of cancer cells that can self-renew and differentiate into various cell types, are responsible for tumor initiation, recurrence, and metastasis [1,2]. The existence of CSCs is considered to be a cause of drug-resistant cancers, since they eliminate sources of biochemical stress. Numerous studies have been performed to isolate CSCs from other cancer cells, and several significant markers have been identified for some cancers. Importantly, the expression of several CSC markers has been reported to enhance tumor progression and maintain a malignant phenotype.

CD44 is one of the most famous CSC markers for gastrointestinal cancer. Several studies have demonstrated that CD44 enhances the malignant phenotype. High expression levels of CD44 are significantly correlated with both tumor size and invasiveness $[3,4]$. The expression of a splice variant of CD44 (CD44v) is frequently associated with the progression of colon and gastric cancers [5-7]. The multifunctional roles of CD44 in the microenvironment and in the regulation of cancer stemness, as well as the demonstrated prognostic value of CD44 for various cancers, suggest that targeting CD44 could be a promising approach with the potential to eliminate CSCs.

In CSCs, reactive oxygen species (ROS) are kept at low levels. ROS play an important role in the formation of a proliferation-permissive intracellular environment and the preservation of a self-renewal capacity $[8,9]$. In cancer tissues, a high ROS level is counteracted by antioxidant enzymes that are produced by cancer cells or inflammatory cells [10]. Although ROS produce oxidative stress in most cancer cells, CSCs possess enhanced mechanisms for protecting against such stress induced by ROS. One of the main mechanisms is glutathione metabolism. CD44v isoforms interact with and stabilize xCT (SLC7A11), a subunit of the cystine/glutamate antiporter known as system $\mathrm{xc}(-)$. The expression of $\mathrm{xCT}$ on the cell surface is essential for the uptake of cystine, which is required for intracellular glutathione (GSH; a major antioxidant), and thereby potentiates the ability of cancer cells to increase glutathione synthesis and defend themselves against ROS [11-13]. This mechanism is reportedly associated with not only drug resistance, but also the generation and growth of tumors. The ablation of CD44 induced the loss of xCT from the cell surface and suppressed tumor growth in a transgenic mouse model of gastric cancer [12].

Despite accumulating evidence regarding CD44-targeting strategies for CSCs, the promised agents have not yet been established [14,15]. 5Aminosalicylic acid (5-ASA), the most commonly used antiinflammatory medication for inflammatory bowel diseases (IBD), has been reported to reduce the risk of CRC significantly $[16,17]$. Since chronic inflammation is a key factor in carcinogenesis, the effect of 5ASA therapy on reducing the risk of CRC may be mediated by a reduction in mucosal inflammation. Recent studies have revealed that sulfasalazine, one of the 5-ASA drugs, might have an even more important role. Sulfasalazine consists of sulfapyridine and the active moiety 5-ASA that are released in the gut. Intact sulfasalazine, but not its metabolites, was found to inhibit the cystine/glutamate transporter (Figure 1) [18]. As a result, GSH synthesis can be efficiently suppressed by the absence of intracellular cystine [19]. Several studies have also shown the function of sulfasalazine as a specific inhibitor of xCTmediated cystine transport and its ability to inhibit the growth, invasion, and metastasis of several types of cancer $[11,18,20]$. We have recently shown that in a mouse experiment, sulfasalazine selectively induced apoptosis in CD44v-expressing head and neck cancer cells and promoted the sensitivity of tumors to anti-cancer drugs [21]. Additionally, in a gastric cancer mouse model, sulfasalazine treatment suppressed the development of precancerous lesions and cancer [22]. Therefore, sulfasalazine might be a promising agent targeting CD44vpositive CSCs for the prevention and treatment of CSCs.

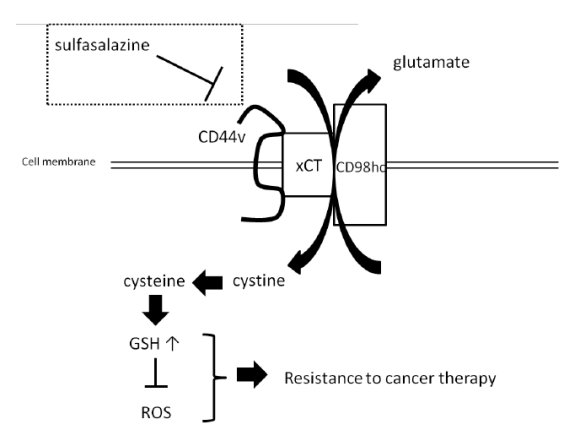

Figure 1: Scheme for CD44-targeting therapy using sulfasalazine (modified from Reference 12); System xc(-) is a cystine/glutamate exchange transporter composed of a light-chain subunit (xCT) and a heavy-chain subunit (CD98hc); CD44v maintains a high level of intracellular GSH by stabilizing the expression of $\mathrm{xCT}$ at the plasma membrane, and thereby associates with resistance to cancer therapy; Sulfasalazine is an agent which inhibits this cystine/ glutamate transporter system.

Based on accumulated evidence, we conducted a retrospective review of the records of ulcerative colitis (UC)-associated CRC patients who underwent curative colorectal surgery [23]. We then compared the patients' clinicopathological findings according to the length of sulfasalazine administration. As a result, long-term sulfasalazine administration was shown to reduce proliferative CD44v9-positive 
cells in cancer and increase the degree of adenocarcinoma differentiation. Furthermore, an in vitro assay revealed that sulfasalazine promoted the expression of epithelial differentiation markers (E-cadherin and CDX2) and inhibited the proliferation of CD44-positive cancer cells. These findings indicate the importance of CD44v9-positive cells in UC-associated cancer progression and differentiation, suggesting that sulfasalazine may serve as a novel therapeutic agent that targets CD44v9-positive cells. There were some limitations in this study: a small number of patients, not considering the dose intensity of sulfasalazine, and the limitation of patients to UCassociated cancer patients. Therefore, further study is needed to investigate whether sulfasalazine affects sporadic cancers as well.

Consistent with previous observations, CD44 is considered to be an ideal target for the development of clinical therapeutics against CSCs. However, only a few clinical trials targeting CD44 have been conducted so far, and none of these trials have resulted in an effective treatment [14]. The limitations demonstrated in previous clinical trials examining anti-CD44 therapies highlight the need for the development of improved anti-CD44v reagents, such as high-affinity anti-CD44v peptides, to replace highly immunogenic antibodies [24] as well as the use of CSC-specific CD44v peptides for cancer vaccines based on promising CD44 DNA vaccine studies in animal models $[25,26]$.

\section{Conclusion}

In conclusion, targeting CD44 is a promising approach with the potential to eliminate CSCs. Better understanding of the fundamental characteristics of CD44 and improvements in drug development targeting CD44 should bring new hope to patients with lifethreatening cancers.

\section{References}

1. Merlos-Suárez A, Barriga FM, Jung $\mathrm{P}$, Iglesias $\mathrm{M}$, Céspedes $\mathrm{MV}$, et al. (2011) The intestinal stem cell signature identifies colorectal cancer stem cells and predicts disease relapse. Cell Stem Cell 8: 511-524.

2. Oskarsson T, Batlle E, Massagué J (2014) Metastatic stem cells: sources, niches, and vital pathways. Cell Stem Cell 14: 306-321.

3. Choi D, Lee HW, Hur KY, Kim JJ, Park GS, et al. (2009) Cancer stem cell markers CD133 and CD24 correlate with invasiveness and differentiation in colorectal adenocarcinoma. World J Gastroenterol 15: 2258-2264.

4. Bendardaf R, Elzagheid A, Lamlum H, Ristamäki R, Collan Y, et al. (2005) E-cadherin, CD44s and CD44v6 correlate with tumour differentiation in colorectal cancer. Oncol Rep 13: 831-835.

5. Tanabe KK, Ellis LM, Saya H (1993) Expression of CD44R1 adhesion molecule in colon carcinomas and metastases. Lancet 341: 725-726.

6. Mayer B, Jauch KW, Günthert U, Figdor CG, Schildberg FW, et al. (1993) De-novo expression of CD44 and survival in gastric cancer. Lancet 342: 1019-1022.

7. Miwa T, Watanabe A, Yamada Y, Shino Y, Yamada T, et al. (1996) Progression in Gastric Carcinoma Relative to the Ratio of CD44 Epithelial Variant Transcript to CD44 Hematopoietic Variant Transcript. Cancer 77: 25-9.
8. Jang YY, Sharkis SJ (2007) A low level of reactive oxygen species selects for primitive hematopoietic stem cells that may reside in the low-oxygenic niche. Blood 110: 3056-3063.

9. Zhou D, Shao L, Spitz DR (2014) Reactive oxygen species in normal and tumor stem cells. Adv Cancer Res 122: 1-67.

10. Warburg O (1956) On the origin of cancer cells. Science 123: 309-314.

11. Lo M, Wang YZ, Gout PW (2008) The $\mathrm{x}(\mathrm{c})$-cystine/glutamate antiporter: a potential target for therapy of cancer and other diseases. J Cell Physiol 215: 593-602.

12. Ishimoto T, Nagano O, Yae T, Tamada M, Motohara T, et al. (2011) CD44 variant regulates redox status in cancer cells by stabilizing the $\mathrm{xCT}$ subunit of system $\mathrm{xc}(-)$ and thereby promotes tumor growth. Cancer Cell 19: 387-400.

13. Nagano O, Okazaki S, Saya H (2013) Redox regulation in stem-like cancer cells by CD44 variant isoforms. Oncogene 32: 5191-5198.

14. Zöller M (2011) CD44: can a cancer-initiating cell profit from an abundantly expressed molecule? Nat Rev Cancer 11: 254-267.

15. Orian-Rousseau V, Ponta H (2015) Perspectives of CD44 targeting therapies. Arch Toxicol 89: 3-14.

16. van Staa TP, Card T, Logan RF, Leufkens HG (2005) 5-Aminosalicylate use and colorectal cancer risk in inflammatory bowel disease: a large epidemiological study. Gut 54: 1573-1578.

17. Velayos FS, Terdiman JP, Walsh JM (2005) Effect of 5-aminosalicylate use on colorectal cancer and dysplasia risk: a systematic review and metaanalysis of observational studies. Am J Gastroenterol 100: 1345-1353.

18. Gout PW, Buckley AR, Simms CR, Bruchovsky N (2001) Sulfasalazine, a potent suppressor of lymphoma growth by inhibition of the $\mathrm{x}(\mathrm{c})$-cystine transporter: a new action for an old drug. Leukemia 15: 1633-1640.

19. Diehn M, Cho RW, Lobo NA, Kalisky T, Dorie MJ, et al. (2009) Association of reactive oxygen species levels and radioresistance in cancer stem cells. Nature 458: 780-783.

20. Chen RS, Song YM, Zhou ZY, Tong T, Li Y, et al. (2009) Disruption of xCT inhibits cancer cell metastasis via the caveolin-1/beta-catenin pathway. Oncogene 28: 599-609.

21. Yoshikawa M, Tsuchihashi K, Ishimoto T, Yae T, Motohara T, et al. (2013) $\mathrm{xCT}$ inhibition depletes CD44v-expressing tumor cells that are resistant to EGFR-targeted therapy in head and neck squamous cell carcinoma. Cancer Res 73: 1855-1866.

22. Wada T, Ishimoto T, Seishima R, Tsuchihashi K, Yoshikawa M, et al. (2013) Functional role of CD44v-xCT system in the development of spasmolytic polypeptide-expressing metaplasia. Cancer Sci 104: 1323-1329.

23. Seishima R, Okabayashi K, Nagano O, Hasegawa H, Tsuruta M, et al. (2016) Sulfasalazine, a therapeutic agent for ulcerative colitis, inhibits the growth of CD44v9+ cancer stem cells in ulcerative colitis-related cancer. Clin Res Hepatol Gastroenterol .

24. Park HY, Lee KJ, Lee SJ, Yoon MY (2012) Screening of peptides bound to breast cancer stem cell specific surface marker CD44 by phage display. Mol Biotechnol 51: 212-220.

25. Wallach-Dayan SB, Rubinstein AM, Hand C, Breuer R, Naor D (2008) DNA vaccination with CD44 variant isoform reduces mammary tumor local growth and lung metastasis. Mol Cancer Ther 7: 1615-1623.

26. Parmiani G, Russo V, Maccalli C, Parolini D, Rizzo N, et al. (2014) Peptidebased vaccines for cancer therapy. Hum Vaccin Immunother 10: 3175-3178. 